\title{
POTENTIAL AND LIMITATIONS OF PHOTOMETRIC RECONSTRUCTION THROUGH A FLOCK OF DOVE CUBESATS
}

\author{
B. Altena ${ }^{a *}$ A. Mousivand ${ }^{b}$, J. Mascaro ${ }^{c}$, A. Kääb ${ }^{a}$ \\ ${ }^{a}$ Department of Geosciences, University of Oslo, 0371 Oslo, Norway - (bas.altena, kaeaeb)@ geo.uio.no \\ ${ }^{\mathrm{b}}$ Remote Sensing and GIS department, Tarbiat Modares University, 14115-111 Tehran, Iran - a.mousivand@ modares.ac.ir \\ ${ }^{\mathrm{c}}$ Planet, San Francisco, 94103, USA - joe.mascaro@ planet.com
}

\section{Commission III, WG III/1}

KEY WORDS: photometry, cubesats, topographic reconstruction, cryosphere, reflection retrieval

\begin{abstract}
:
When Earth observation satellite systems are designed, one typically prefers a sun-synchronous orbit. However, the first generations of cubesats from Planet were deployed out of the International Space Station (ISS) and therefore do not obey such an orbit. Their configuration samples at different local times within the mid-latitudes. Consequently, it is in theory possible to exploit photometric techniques and extract highly detailed topographic information. In this study we demonstrate and explore photometry based on Planet cubesat images for Tyndall glacier at the Southern Patagonian icefield, and Zhadang glacier situated on the Tibetan plateau.
\end{abstract}

\section{INTRODUCTION}

Since its introduction in the 1980's (Woodham, 1980, Brooks and Horn, 1985), photometric stereo has advanced into a well developed field of science within computer vision. Current research is focused on subjects with non-Lambertian reflection and/or unknown lighting (Ackermann and Goesele, 2015). In Earth observation the ill-posed formulation of photometry, photoclinometry, has been applied (Peng et al., 2015). Though its applications have mostly been concentrated around snow-covered cryospheric phenomena, such as glacier topography (Scambos and Fahnestock, 1998, Altena, 2012, Pope et al., 2013), streaklines (Raup et al., 2005), icedolines (Bindschadler et al., 2002), or subglacial lake drainage (Fricker et al., 2010). The bias towards cryospheric applications might stem from the fact that the reflection behavior of snow in the visible spectrum is close to isotropic (Dumont et al., 2010). Other advanced reflection functions have been applied in Earth observation, however then the photometric configuration is reversed; illumination is set fixed and the acquisition positions change (Heipke et al., 2001)

The absence of mainstream implementations for photometric techniques within the Earth observation community stems from the (for this purpose) unfavorable orbital configuration of Earth observation satellites. Preferably, these satellites are set into a sunsynchronous orbit, where the revisit is at the same local time, hence collecting imagery with an almost fixed sun azimuth. Only at high latitudes Earth observation satellites like Sentinel-2 or Landsat8 have different sensing times due to orbital cross-over (Kääb et al., 2016). Daily sampling is possible with a system such as MODIS, however this platform has low resolution instruments (250-1000 meters). The Earth observation community is well aware of the photometric principles and bi-directional reflection functions (BRDF) are well established within its field. The lack of use of photometry is mostly because the topography is assumed to be known, through digital elevation models

\footnotetext{
${ }^{*}$ Corresponding author: Bas Altena (bas.altena@geo.uio.no)
}

(DEM) from photogrammetry, laser scanning or radar interferometry. However, with the now-emerging high resolution satellites with different acquisitions at different times of the day, photometry becomes in theory feasible. This technique can, for instance, be of use for analysis of processes at the Earth surface that change topography. In this study we explore the possibility of photometry, through direct estimation of topography without atmospheric correction over a section of Tyndall glacier, at the Southern part of the Southern Patagonian Icefield. We also explore the possibilities of topographic reconstruction with atmospherically corrected imagery over complex terrain. The glacier for this experiment is situated within the remote mountain region of Nyainqêntanglha on the Tibetan plateau. The purpose of this study is not to come up with a new approach to estimate Earth topography, but rather to discuss potential and limitations of Earth observation satellitebased photometry by means of the rare opportunity offered by the Planet cubesat constellation.

\section{BACKGROUND}

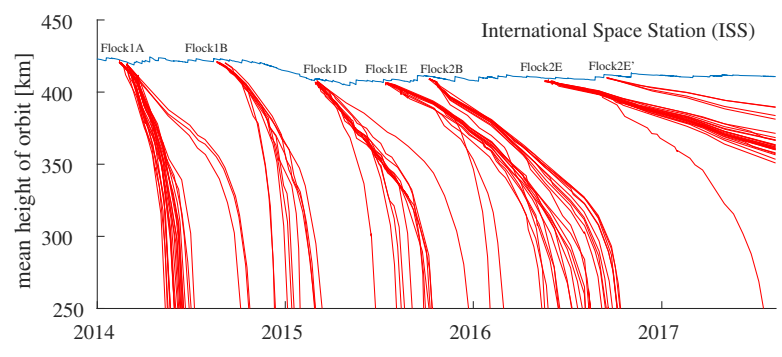

Figure 1. Orbital decay of the ISS in blue and different generations of Planet cubesats (called Doves) deployed from it. For every Dove the orbit altitude plotted.

\subsection{PlanetScope constellation}

The objective of the Plant constellation is to observe the Earth surface every day, with a pixel resolution of 3-5 meters in the visible and near-infrared spectrum. This is achieved with cubesats, 
or Doves, equipped with a standard CCD chip, having a field-ofview on the Earth surface of 20-30 kilometer (Planet, 2017, Kääb et al., 2017). Consequently, a multitude of cubesats is needed in order to achieve this goal. At the time of writing, the planet constellation consists of two different orbits, a sun-synchronous and a low-Earth orbit. The latter are Doves deployed from the ISS, and consists of the first dozen of cubesats launched by Planet. A specific feature of this constellation concept is the amount of change in design, every flock serves as a test bed for the next flock of cubesats to be produced (Boshuizen et al., 2014). Consequently, specifications change as is observable for example in figure 1, where the effective drag of the cubesats within the upper atmosphere seems to decrease launch after launch. Another example of this concept is the near-infrared (NIR) band which is now included in newer generations of Doves.

For the ISS orbit, specifically, the batch deployment of cubesats, called a flock, was done over the course of several days or weeks. The closer to the Earth surface, the more drag the cubesats will experience from the atmosphere. This drag is proportional to the cross-section of the object. This orbital decay is in the order of two kilometers per month for the ISS. To stay in its nominal orbit of roughly 420 kilometers above the Earth, thrusters boost the station when needed. However, such thrusters are absent for the cubesats of Planet, thus after some months they burn up in the upper atmosphere, as can be seen in figure 1 .

Due to a time lag of deployment from the ISS, the already launched cubesats decay slower, thus generating a spread within the orbit. Consequently, a flock of Doves follows a similar orbit as the ISS, thus completing one orbit in roughly 92 minutes. On one such orbital circulation, a flock of cubesats travels as illustrated in figure 2 . Here one can see that especially around $\pm 50^{\circ}$ latitude the flock passes through multiple timezones (the vertical axis). This results in a burst of observations, over the coarse of some hours/days at certain locations on the Earth. For weeks the flock senses over other parts of the Earth, after which they return again. It is at these bursts of image collection where photometric techniques can be used for topographic snapshots. In this study we explore the photometric technique over somehow static topography and assess its capabilities.

\subsection{Photometry}

The collected intensities on a photosensitive cell $(I)$ are the result of a chain of components. First, the radiation of the sun $(S)$ comes to the surface where it reflects. The amount of received energy is dependent on the shading, which is an interplay between the surface normal $(\vec{n})$ and the sun direction $(\vec{s})$, both vectors having unit length. Because of the isotropy assumption we adopt here, the reflectance $(R)$ can be seen as a constant in time and space. Furthermore for simplicity of formulation, we exclude just for now in-path absorption, however for a full account see (Mousivand et al., 2015). In this simple formulation the sensor has a certain transmittance $(T)$, where after the energy over a certain bandwidth over different wavelengths $(\lambda)$ is collected and transformed $(C)$ to a digital number. The sensor sensitivity has a lower bound $\left(L_{0}\right)$ and $(A)$ denotes indirect radiance. Furthermore, sensor noise is excluded for sake of simplicity in this formulation, and the energy collection can then be stated as (Bindschadler and Vornberger, 1994),

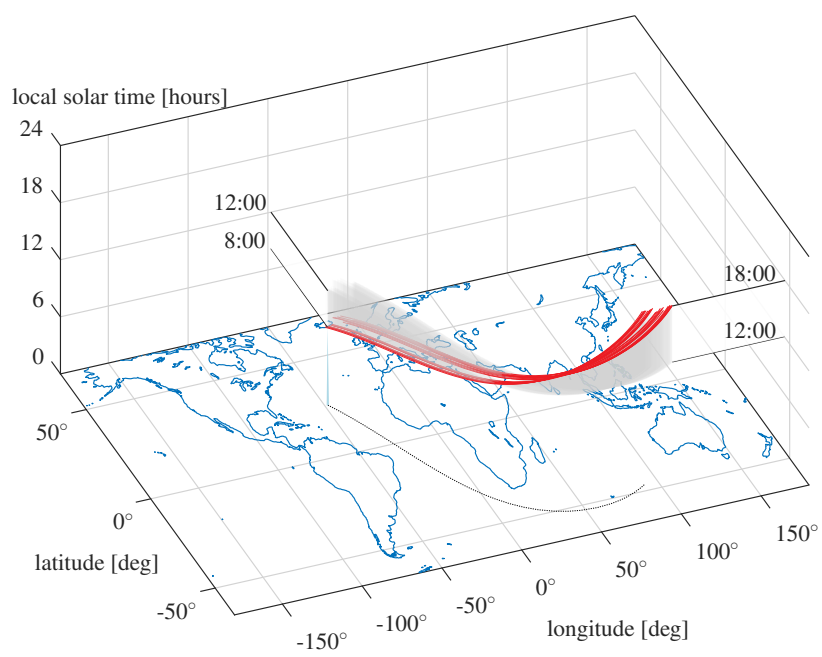

Figure 2. The spatial-temporal fly-through of one Planet flock in ISS-orbit. The paths are in respect to local-time as the flock flies through a nominal day on Earth.

$$
I=C \int_{\lambda} T(\lambda)\left[S(\lambda) R(\lambda) \vec{n} \cdot \vec{s}+A(\lambda)-L_{0}(\lambda)\right] d \lambda .
$$

These components can be estimated over the given spectral interval and their discretized version is the mean over this interval, denoted by an overbar. This transforms the integral to constants and equation 1 simplifies to,

$$
I=\bar{C} \bar{T}\left[\bar{S} \bar{R} \vec{n} \cdot \vec{s}+\bar{A}-\bar{L}_{0}\right]
$$

In such a case, the atmospheric and sensor terms can be removed, and the leftover can be rearranged into a simple linear function where most of the components can be put into one term $(\kappa)$. Then, for multiple acquisitions $(n)$ the following system of equations can be formulated, following (Coleman and Jain, 1982);

$$
\underbrace{\left[\begin{array}{c}
I_{1} \\
\vdots \\
I_{n}
\end{array}\right]}_{\mathbf{y}}=\underbrace{\left[\begin{array}{c}
\vec{s}_{1}^{\top} \\
\rightarrow \\
\vdots \\
\vec{s}_{n}^{\top}
\end{array}\right]}_{\mathbf{L}} \underbrace{\kappa \vec{n}}_{\mathbf{x}}+\underbrace{\left[\begin{array}{c}
A_{1} \\
\vdots \\
A_{n}
\end{array}\right]}_{\nabla} .
$$

The unknown sensor and normal term, within $\mathbf{x}$, can be estimated through least squares estimation from the intensity vector $(\mathbf{y})$ and sun-configurations $(\mathbf{L})$ matrix. The decomposition is done through normalization, $(\kappa=\|\mathbf{x}\|)$, in order to make the surfacenormal a unit vector:

$$
\left[\begin{array}{c}
x \\
\nabla
\end{array}\right]=\left([\mathbf{L} \mid \mathbf{I}]^{\top}[\mathbf{L} \mid \mathbf{I}]\right)^{-1}[\mathbf{L} \mid \mathbf{I}]^{\top} \mathbf{y}, \quad \vec{n}=\mathbf{x} /\|\mathbf{x}\|
$$

\section{RADIOMETRIC PROCESSING}

Since the production of Flock 2E, the Dove satellites are calibrated while in orbit. Before launch the photo-sensitivity and 


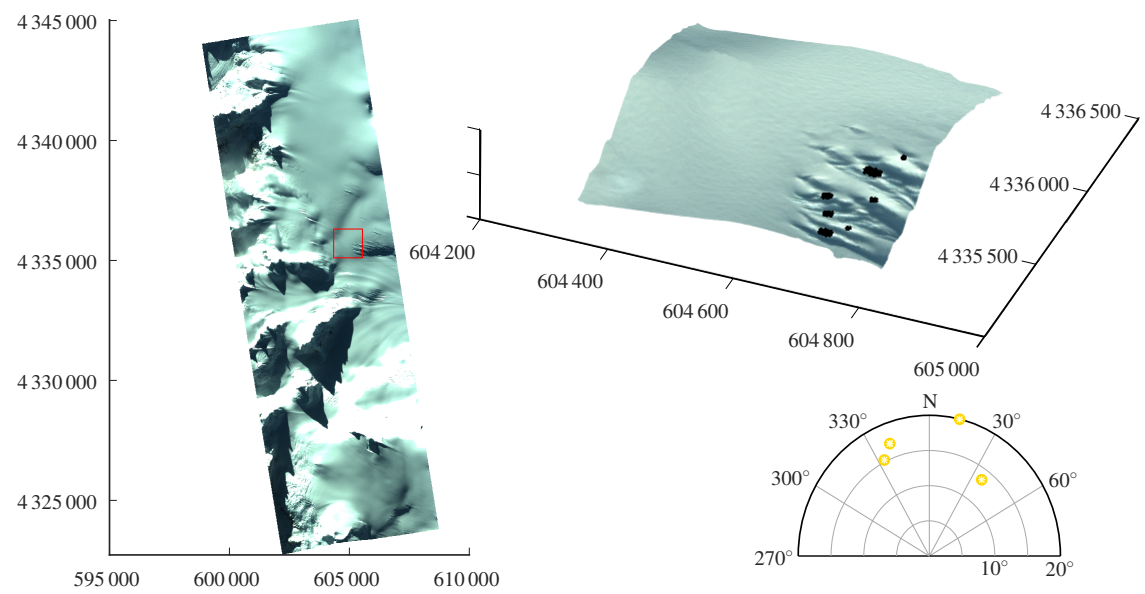

Figure 3. Left) A planetScope acquisition over the upper part of Tyndall-Glacier. Upper right) The estimate surface model is derived from the section outlined in red, on the left panel. The planar axis in this panel is in meters (UTM), while the vertical axis is a relative scale. Lower right) Polar plot of the sun angles of the imagery used for the reconstruction.

color filter transmittance are used to formulate the relative spectral response (RSR). For every flock from $2 \mathrm{E}$ onwards such spectral response curves are used to cross-calibrate every satellite with Landsat 8 or the RapidEye constellation and through sensing the lunar surface (Wilson et al., 2017).

These given spectral response curves $(C(\lambda))$ serve in the present study as input for a MODTRAN-based atmospheric correction estimation (Mousivand et al., 2015). For this atmospheric modeling only a small amount of parameters is needed (water vapor, visibility and $\mathrm{CO}_{2}$ mixing). In the case of Zhadang glacier MODIS products where used to extract these parameters.

Before any calculations, one is able to analyze the potential accuracy of the estimate and the measurement design of the sunconfigurations ( $\mathbf{L})$. This can be done through error propagation, following (Teunissen, 2003, Hold-Geoffroy et al., 2015):

$$
\vec{\delta}=\frac{1.96 \sigma}{R} \operatorname{diag}\left(\mathbf{L}^{\top} \mathbf{L}\right)^{-1}
$$

For simplicity the atmospheric effects are neglected in this configuration. The other parameters can be estimated, for example, the reflection $(R)$ is in the visible range within 0.8-0.9 and in the NIR around 0.7-0.8, depending on the freshness of the snow (Warren, 1982). Furthermore, the radiometric precision $(\sigma)$ is around 5\% accurate (Wilson et al., 2017). Hence with these numbers it is possible to estimate the potential accuracy of the normal vectors. Unfortunately, from this analysis it came to light that for both case studies the design matrix is not well conditioned For example, Zhadang glacier is only well posed in the azimuth: $\vec{\delta}=\left[\begin{array}{lll}0.1 & 3.6 & 7.4\end{array}\right]^{\top}$.

\section{RESULTS AND DISCUSSION}

Our first case study covers a part of the icefield of Tyndall glacier $\left(51.0^{\circ} \mathrm{S} 73.5^{\circ} \mathrm{W}\right)$, which is situated at the southern tip of the Southern Patagonian Icefield. A total of 6 Planet cubesat image strips were included, each composed of only three bands (red, green and blue). The acquisition time of the Planet data spread from 9:36 until 14:00 local time, between $24^{\text {th }}$ and $31^{\text {th }}$ of July 2016. Converting to solar angles, this relates to a spread in azimuth of $36^{\circ}-332^{\circ}$, while the zenit angle is between $13^{\circ}$ and $20^{\circ}$. The acquisition information is illustrated in figure 3 . In this plot it becomes clear that the sun angles do not describe a plane, but a cone (Shen et al., 2014) as it is close to solstice. Nevertheless, this configuration is not optimal as the low sun elevation make atmospheric transfer modeling unreliable. Therefore, in this case study we used the raw intensity values and put all terms $\left(C, S, A, L_{0}\right)$ into one parameter $(\kappa)$, neglecting indirect reflection and adjacency effects $(\nabla)$. The red band in all images was used, as this band has the lowest reflection for snow, hence the least saturation. The estimated topography is illustrated in figure 3 , which is an integration of the normals into an elevation model and done within the frequency domain (Frankot and Chellappa, 1988).

In general the topography of the crevasses are extracted from the intensities. A low frequency bulge is present, but this stems from the integration based on continuous series. Also in some places there are black spots (voids). This is where one of the sensors had been saturated, and it was not possible to estimate the normal. This saturation comes from the high reflectivity of snow in the visible range, though this reflection drops in the NIR.

The second case study is for the Zhadang glacier, a small mountain glacier in the Nyainqêntanglha mountain range, on the Tibetan plateau. The imagery was collected between the $11^{\text {th }}$ and $16^{\text {th }}$ of December 2016, with varying sun angles. The imagery was transformed to near surface reflectance values without estimating reflectance contributed from other terrain (Mousivand et al., 2015). However, the indirect reflection or adjacency term $(A)$ was assumed to be stable within a small region, thus when estimating, the topography of a template is taken and the atmospheric adjacency effect seen as a local constant bias (see equation 4). Because only the reflection $(R)$ is not set in this configuration, the estimated albedo $(\kappa)$ should be a similar value.

For validation, the ALOS World 3D DEM (AW3D30), a 30 meter resolution digital surface model (Tadono et al., 2014), is used. The estimated azimutal component from this surface model and the photometric estimates are illustrated in figure 4. Several ef- 


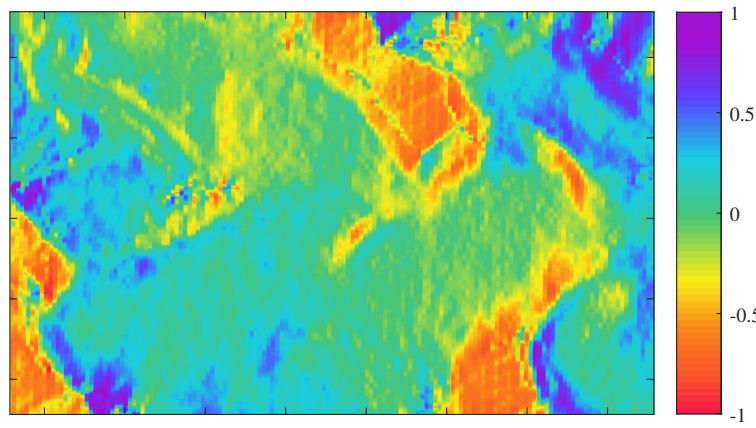

(a) azimuthal vector component $\left(\vec{n}_{x}\right)$ from photogrammetric DEM

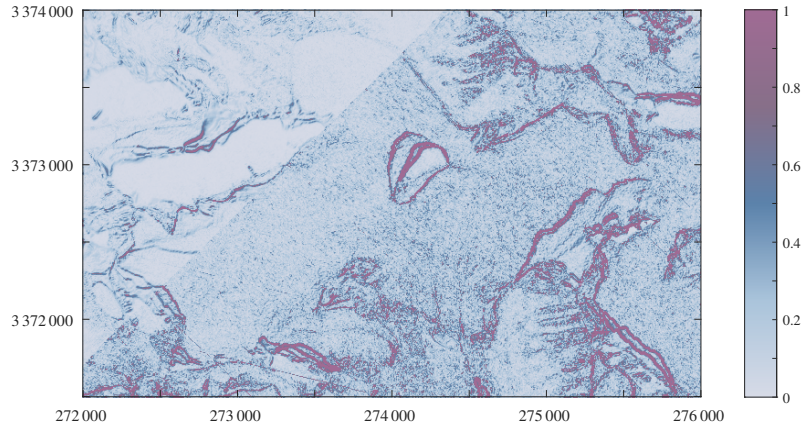

(c) normal vector component $(\kappa)$ from photometric DEM

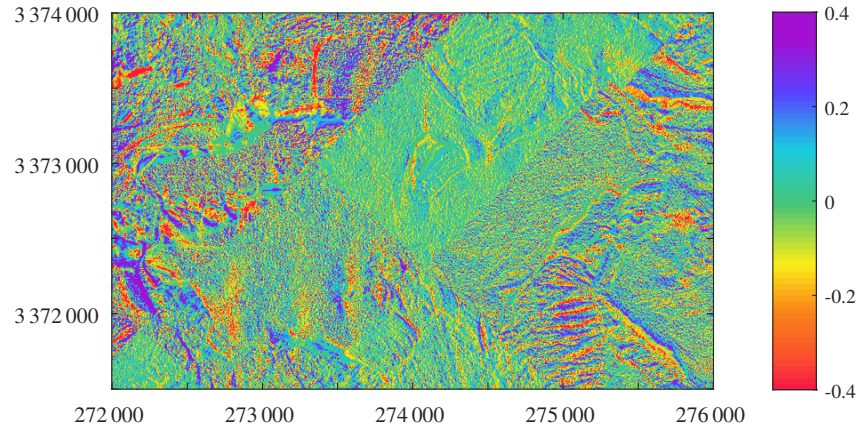

(b) azimuthal vector component $\left(\vec{n}_{x}\right)$ from photometric DEM

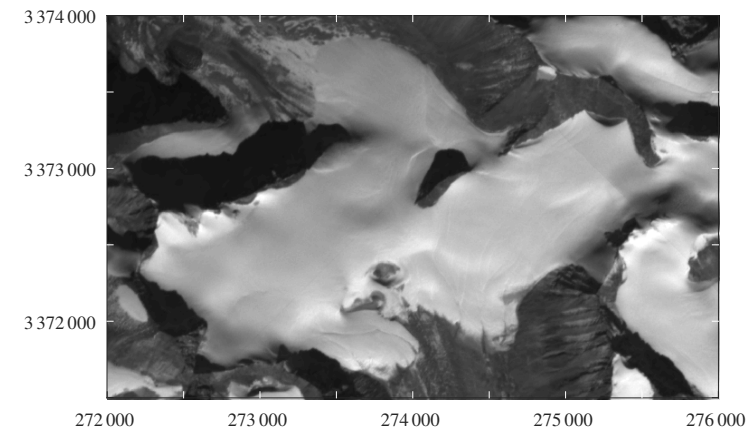

(d) NIR acquisition $(I)$ from Planet Dove

Figure 4. Topographic normals of the photogrammetric DEM (validation), photometric and albedo product (estimate) and acquisition (input) over Zhadang glacier $\left(30.5^{\circ} \mathrm{N} 90.6^{\circ} \mathrm{E}\right)$.

fects can be seen, firstly the estimated normal is not in the correct range. Secondly, biases are apparent within block like shapes. These stem from the size of the images used, as not every image strip covers the full extent of the study site. The offset in the estimate suggests a bias within the input data, either from the atmospheric transfer modeling or the sensor calibration. Nevertheless, topographic signals on the glacier are picked up, such as the undulations at the glacier snout. However, the signals are noisy mainly seem to reflect the higher frequency signals. Furthermore, within the imagery itself radiometric jumps occur, as can be seen in atmospherically corrected image. This would indicate that the radiometric calibration is not consistent between imagery. This can be due to the fact that calibration is done for the full frame and not specifically per pixel. Lastly, the estimated albedo is a good proxy if all atmospheric terms are isolated. In the ideal case, this parameter should have a value of around 0.7. However, as can be seen, this is certainly not the case for shadowed areas. But also the majority of albedo values are too low for a snow surface. This would hint towards some inabilities of the radiative transfer modelling to describe the atmosphere.

\section{CONCLUSION AND PERSPECTIVES}

The Planet Doves in ISS orbit where not intentionally positioned into a photometric constellation. However, this study shows topographic information is present and to some extent extractable from such snapshots, but limitations clearly exist. When using imagery over high latitudes and close to the solstice, the available imagery will have a suitable configuration of sun angles. However, this will result in low sun angles, which make in turn atmospheric modelling troublesome. On the other hand, when terrain is estimated at lower latitudes, the sun angles get within a plane, which then decreases the suitability of the configuration for photometry. In addition, a pronounced bias is present in our results, which could come from calibration of the data used or an inability in our model to isolate every atmospheric contribution.

Photometry is a not a well explored method within Earth observation. Nevertheless, the highlighted configuration and its relation to atmospheric modeling opens up further directions into better understanding radiative transfer modeling in complex terrain. Because the reflection function of snow is comparably simple, this relation can be used to improve and test such modeling of complex terrain. If fruitful, this method would then open the door for the full exploitation of photometry and its integration with photogrammetric processing. Hence, photometry and the configuration of Planet could have the potential to transform imagery to operational products with high spatio-temporal content when combined with other photogrammetric systems.

\section{ACKNOWLEDGMENT}

The authors would like to thank Planet for providing the data through the Science Ambassador program and support from the European Union FP7 ERC project ICEMASS (320816) and the ESA project Glaciers_cci (4000109873 14 I-NB). Bas Altena designed the study, performed most analysis, and wrote the paper. Alijafar Mousivand provided and wrote the code for the radiative transfer modeling. Joseph Mascaro provided the data and support, he as well edited the paper. Andreas Kääb contributed to discussions and edited the paper.

Conflict of interest Bas Altena, Alijafar Mousivand and Andreas Kääb declare no competing interests. Joseph Mascaro is program manager for impact initiatives at Planet. He did in no manner influence the results or conclusions of the study. 


\section{REFERENCES}

Ackermann, J. and Goesele, M., 2015. A survey of photometric stereo techniques. Foundations and Trends $\mathrm{R}$ in Computer Graphics and Vision 9(3-4), pp. 149-254.

Altena, B., 2012. Filling the white gap on the map: Photoclinometry for glacier elevation modelling. Master's thesis, TU Delft, Delft University of Technology.

Bindschadler, R. and Vornberger, P., 1994. Detailed elevation map of ice stream C, Antarctica, using satellite imagery and airborne radar. Annals of glaciology 20, pp. 327-335.

Bindschadler, R., Scambos, T., Rott, H., Skvarca, P. and Vornberger, P., 2002. Ice dolines on Larsen ice shelf, Antarctica. Annals of glaciology 34(1), pp. 283-290.

Boshuizen, C., Mason, J., Klupar, P. and Spanhake, S., 2014. Results from the Planet labs flock constellation. In: Proceedings of 28th annual AIAA/USU conference on small satellites, SSC14, Vol. 1number I, pp. 1-8.

Brooks, M. and Horn, B., 1985. Shape and source from shading. In: International joint conference on artificial intelligence, pp. 932-936.

Coleman, E. and Jain, R., 1982. Obtaining 3-dimensional shape of textured and specular surfaces using four-source photometry. Computer graphics and image processing 18(4), pp. 309-328.

Dumont, M., Brissaud, O., Picard, G., Schmitt, B., Gallet, J. and Arnaud, Y., 2010. High-accuracy measurements of snow bidirectional reflectance distribution function at visible and NIR wavelengths-comparison with modelling results. Atmospheric chemistry and physics 10, pp. 2507-2520.

Frankot, R. and Chellappa, R., 1988. A method for enforcing integrability in shape from shading algorithms. IEEE Transactions on pattern analysis and machine intelligence 10(4), pp. 439-451.

Fricker, H., Scambos, T., Carter, S., Davis, C., Haran, T. and Joughin, I., 2010. Synthesizing multiple remote-sensing techniques for subglacial hydrologic mapping: Application to a lake system beneath MacAyeal ice stream, west Antarctica. Journal of glaciology 56(196), pp. 187-199.

Heipke, C., Piechullek, C. and Ebner, H., 2001. Simulation studies and practical tests using multi-image shape from shading. ISPRS journal of photogrammetry and remote sensing 56(2), pp. 139-148.

Hold-Geoffroy, Y., Zhang, J., Gotardo, P. and Lalonde, J.-F., 2015. What is a good day for outdoor photometric stereo? In: IEEE International Conference on Computational Photography, IEEE, pp. 1-9.

Kääb, A., Altena, B. and Mascaro, J., 2017. Coseismic displacements of the 14 November 2016 Mw 7.8 Kaikoura, New Zealand, earthquake using the Planet optical cubesat constellation. Natural Hazards and Earth System Sciences 17(5), pp. 627.

Kääb, A., Winsvold, S., Altena, B., Nuth, C., Nagler, T. and Wuite, J., 2016. Glacier remote sensing using Sentinel-2. Part I: Radiometric and geometric performance, and application to ice velocity. Remote sensing 8(7), pp. 2072-4292.

Mousivand, A., Verhoef, W., Menenti, M. and Gorte, B., 2015. Modeling top of atmosphere radiance over heterogeneous nonlambertian rugged terrain. Remote Sensing 7(6), pp. 8019-8044.

Peng, J., Zhang, Y. and Shan, J., 2015. Shading-based DEM refinement under a comprehensive imaging model. ISPRS Journal of photogrammetry and remote sensing 110, pp. 24-33.

Planet, T., 2017. Planet application program interface: In space for life on earth. San Francisco, CA.

Pope, A., Willis, I., Rees, W., Arnold, N. and Pálsson, F., 2013. Combining airborne LiDAR and Landsat ETM+ data with photoclinometry to produce a digital elevation model for Langjökull, Iceland. International journal of remote sensing 34(4), pp. 10051025 .
Raup, B., Scambos, T. and Haran, T., 2005. Topography of streaklines on an Antarctic ice shelf from photoclinometry applied to a single advanced land imager (ALI) image. IEEE transactions on geoscience and remote sensing 43(4), pp. 736-742.

Scambos, T. and Fahnestock, M., 1998. Improving digital elevation models over ice sheets using AVHRR-based photoclinometry. Journal of glaciology 44(146), pp. 97-103.

Shen, F., Sunkavalli, K., Bonneel, N., Rusinkiewicz, S., Pfister, H. and Tong, X., 2014. Time-lapse photometric stereo and applications. In: Computer graphics forum, Vol. 33number 7, Wiley Online Library, pp. 359-367.

Tadono, T., Ishida, H., Oda, F., Naito, S., Minakawa, K. and Iwamoto, H., 2014. Precise global DEM generation by ALOS PRISM. ISPRS Annals of the Photogrammetry, Remote Sensing and Spatial Information Sciences 2(4), pp. 71.

Teunissen, P., 2003. Adjustment theory. Series on Mathematical Geodesy and Positioning, VSSD, Delft, the Netherlands.

Warren, S., 1982. Optical properties of snow. Reviews of geophysics and space physics 20(1), pp. 67-89.

Wilson, N., Greenberg, J., Jumpasut, A., Collison, A. and Weichelt, H., 2017. Absolute radiometric calibration of Planet Dove satellites, Flocks 2p \& 2e. Technical report, Planet Labs \& Planet Berlin GmbH.

Woodham, R., 1980. Photometric method for determining surface orientation from multiple images. Optical engineering 19(1), pp. 139-141. 